\title{
LUXIÉRNAGA
}

Denisse Campos Rodríguez 


\section{La libertad de tener que elegir determinadamente}

\section{Introducción}

El presente escrito es un pequeño esbozo donde se argumentará a favor de la teoría del compatibilismo que David Hume desarrolla en su obra Investigación sobre el conocimiento humano. De este modo, explicaremos las concepciones de libertad, necesidad y causalidad que el filósofo escocés utiliza para la consolidación de su teoría. Aunado a esto, en el primer apartado del ensayo, expondremos los factores que el mismo D. Hume justifica para una verdadera conciliación entre el determinismo y la libertad y, la importancia que tienen estos dos aspectos para la cotidianeidad de las personas, así como él también nos cuestionaremos el por qué, precisamente, se da una confusión entre estas dos nociones. Posteriormente, describiremos la teoría del compatibilismo, del empirista escocés, y cómo es que llega a su afirmación, pero también explicaremos cómo es que las pasiones juegan un papel fundamental en la toma de decisiones del hombre, ya que sin éstas no habría acción.

\section{Factores que intervienen en la conciliación entre determinismo y libertad}

La dicotomía entre libertad y determinismo es un problema que ha estado muy presente a lo largo de toda la historia de la filosofía, a su vez, también resulta una de las cuestiones más discutidas y controversiales dentro de la misma. El tema de la libertad no puede ser minimizado ya que depende de su significación y de las distintas soluciones que se le suscriban para poder describir lo que constituye el hombre en la vida, a partir del supuesto de que la libertad es una característica esencial del ser humano. Si esta facultad, la libertad, resulta ser una falsedad, entonces, el hombre sería simplemente una 
máquina que acata movimientos que ya están determinados, éstos, a su vez, serían la causa de los motivos, creencias o fuerzas que fomentan la realización de cierta acción.

Si nos apegamos a la idea de que el determinismo resulta ser verdadero, tampoco tendría caso el estar evocando méritos, o deméritos, porque sería inútil estarnos lamentando por el mal que causamos o, por el contrario, nuestras alabanzas serían injustas por algún bien que hemos hecho. Otro problema que resultaría de esto es que el sujeto en cuestión nunca podría ser responsable, moralmente, de sus acciones ya que no las realizó por voluntad propia, sino que simplemente lo hizo atendiendo a alguna 'fuerza desconocida' que se lo solicita, o en todo caso, lo obliga a producir sus actos. Es en este sentido que Hume propone su teoría acerca de la libertad y el determinismo y, cómo estas dos posturas pueden ser compatibles, rescatando aspectos como la responsabilidad moral y la necesidad, haciendo una compaginación entre éstos argumentando que hace falta una redefinición de algunos conceptos para que este compatibilismo resulte eficaz.

Se ha pensado que la idea de que las acciones humanas derivan, por necesidad, de sus antecedentes, entra en una problemática con una de las condiciones más importantes de la vida humana: la libertad que, se supone, poseemos todos los seres humanos. Hume tiene muy presente esta tensión e intenta explicar su origen considerando el conflicto que representan estas dos posturas, así como la insatisfacción que a la gente le causa la doctrina de la necesidad y la doctrina de la libertad, estimando que éstas dos posiciones no pueden ser compatibles. Atendiendo a esto, Hume proporciona tres factores que llevan a las personas a suponer, equivocadamente, que ambas cosas no pueden ser conciliadas. El primer factor, nos dice el filósofo, se debe a una confusión o un malentendido; según Hume existen dos tipos de libertad: la 'libertad de espontaneidad' que es aquella que se opone a la violencia y a la coacción; y la 'libertad de indiferencia', que resulta incompatible con la necesidad y las causas, y no diferenciarla del otro tipo de libertad resulta en negar que las acciones humanas sean causadas. La segunda explicación que Hume atribuye a la necedad de la gente por creer que la libertad y necesidad, son 
incompatibles, es la siguiente: se debe a una 'falsa sensación o experiencia' de la ausencia de necesidad o determinación en la acción ${ }^{\mathrm{I}}$, y, por consiguiente, concluimos que ésta no existe. Finalmente, la tercera y última explicación que el filósofo nos brinda para demostrar la inclinación que la gente tiene hacia la doctrina de la libertad, y negar la doctrina de la necesidad, se evoca a la creencia que tienen los individuos con respecto a la religión y la moralidad, creyendo que, si la doctrina de la necesidad resultara ser verdadera, estos dos últimos aspectos implicarían ser meramente ilusorios. La oposición a esta doctrina también se podría especificar más, de tal forma que la necesidad destruye, según Hume, totalmente todo mérito y demérito, ya sea ante la humanidad o ante poderes superiores.

\section{Entre la libertad y la necesidad: compatibilismo humeano}

Es cierto que Hume no ofrece una amplia explicación para el concepto de libertad, ni tampoco explica, plenamente, cómo es que esta concepción pueda "aplicarse consistentemente a acciones que derivan por necesidad de sus antecedentes" ${ }^{2}$. La dilucidación de estas dos cuestiones parecía resolver el conflicto entre la libertad y necesidad, por lo menos tendría grandes posibilidades de conciliarlos, sin embargo, Hume simplemente ignora dichas tareas debido a que él cree que todos debemos adoptar la doctrina de la necesidad para poder asignar la responsabilidad moral y las posibilidades alternativas, y no es solamente que la libertad y la responsabilidad moral sean compatibles con la necesidad, sino que la exigen, sin ella no tendrían sentido ninguno de los dos aspectos.

Por otra parte, Hume cree que es necesaria una nueva redefinición acerca de la libertad y la necesidad, es por esto que proporciona un nuevo concepto para que, de esta forma, la conciliación resulte certera. Es importante señalar que la solución que se da para este compatibilismo tendrá que pasar por el camino que sujeta a la libertad, y a la

\footnotetext{
${ }^{1}$ Stroud, Barry. (1986). Hume, (Trad. Antonio Zirión), UNAM, México, p. 215.

2 Íbidem., p. 219.
} 
necesidad, por el procedimiento que él mismo realiza en su teoría del conocimiento, es decir, el de las impresiones y las ideas. De esta forma, estos dos procesos, el de la reconceptualización y la adjudicación del tratamiento de impresiones e ideas a los términos de libertad y necesidad, permitirá identificar que las nociones comunes que se tenían de estos términos, resultan ilusorias, que lo que se ha dicho de ellas y lo que se funda en ellas resulta irreal y, por lo tanto, las discusiones que se han presentado con respecto a estas cuestiones son inútiles.

Como lo hace Hume, primero atenderemos y examinaremos la doctrina de la necesidad. El filósofo nos dice que universalmente se acepta que toda la materia existente es movida por una 'fuerza' necesaria, es decir, que su curso de acción está determinado, únicamente, por sus causas y que, "ningún otro efecto en estas circunstancias concretas podría resultar de ella" ${ }^{3}$. Dice Hume que, si queremos hacernos una idea correcta acerca de la necesidad, tenemos que considerar de dónde es que surge esta idea cuando la aplicamos a la operación de los cuerpos. Parece evidente, dice Hume, que, si todas las escenas o situaciones de la naturaleza, nunca presentaran esta uniformidad y fueran continuamente cambiadas, de tal manera que cada acontecimiento fuera nuevo y no guardara semejanza con el suceso anterior a él, nunca, de ningún modo, nos hubiésemos formado la idea o noción de necesidad o conexión necesaria. Si este fuera el caso no podríamos más que un hecho es seguido de otro, no que ese hecho fue producido por otro, por consiguiente, no conoceríamos la relación de causa y efecto. Nos dice Hume, por lo tanto, que nuestra idea de necesidad y causación proviene, exclusivamente, de esta uniformidad que se encuentra presente en las operaciones de la naturaleza y que es posible observar las acciones en las que constantemente están unidos objetos similares, "y la mente es llevada por costumbre a inferir uno de ellos de la aparición de otro. Solamente estas dos circunstancias constituyen la necesidad que adscribimos a la

\footnotetext{
${ }^{3}$ Hume, David. (2006). Investigación sobre el conocimiento humano, Alianza, Madrid, p. 106.
} 
materia". Con respecto a la idea de libertad, Hume no proporciona un concepto tan amplio, por lo cual es criticado constantemente.

Para Hume la libertad se fundamenta en poder hacer lo que uno quiere, o desea, y no tener algún impedimento para ejercer dicha acción o tomar una decisión. Más allá de esta concepción, el filósofo no menciona otra característica que sea acreedora de la libertad. Es pertinente comentar que el hecho de que yo carezca de algún impedimento para hacer tal cosa, no significa que la vaya a realizar, porque en eso consisten las posibilidades alternativas.

Lo que el filósofo escocés pretende con esta nueva definición de necesidad, es el poder atribuirla también al comportamiento humano argumentando que, de igual forma, existe una gran uniformidad en las acciones de los hombres, independientemente del lugar en el que se desarrollen, y que la naturaleza humana sigue fiel en lo que respecta a sus principios y operaciones. Lo que quiere decir con esto es que, exactamente, las mismas motivaciones han producido siempre las mismas acciones. Sin embargo, aclara que no esperemos y no supongamos que esta uniformidad de las acciones en los hombres se haga hasta el punto en que todo hombre, en las mismas circunstancias, se conduzca exactamente de la misma manera, ya que existe una gran diversidad de caracteres, prejuicios y opiniones. Ahora, para conjuntar libertad y necesidad, Hume propone que, debido a las experiencias pasadas y tratos que tenemos con otras personas, hemos observado sus comportamientos y nos hemos dado cuenta que actúan de una forma regular; lo mismo sucede con nosotros, cuando realizamos acciones que se suscitan en diferentes momentos y actuamos de forma similar. Es por esto que nosotros albergamos ese hábito esperando que las personas sigan actuando como lo hacen en el pasado, de la misma forma que los elementos exteriores en el mundo. Por su misma naturaleza, las acciones son temporales y efímeras, y si no emergieran de alguna causa, dentro del carácter y disposición de una persona, no podrían colaborar para la alabanza de dicho sujeto si sus acciones resultaran ser moralmente buenas, y tampoco podrían contribuir a

${ }^{4}$ Ibídem, p. 106-107. 
su ultraje si resultaran malas. Estas acciones tienen que provenir, necesariamente, de su voluntad y no de alguna influencia que pudieran tener, por ejemplo, la religión; las decisiones que tome una persona en base a la religión que profesa, al no proceder de la voluntad misma del sujeto, no podría ser acreedor de algún castigo o gloria. No se puede culpar a alguien que realizó algún acto por ignorancia o causalmente. De esta forma, Hume quiere probar que se es libre cuando la acción realizada está de acuerdo con la voluntad, incluso cuando ésta última esté determinada por los motivos o eventos pasados. Aunque la voluntad, de alguna forma, ya se encuentre determinada, la persona todavía puede seguir siendo responsable, moralmente, por las acciones que cometió si las hizo de una forma consciente y si esos motivos, que lo impulsaron a tomar esa decisión, procedieron únicamente de su voluntad. Recordemos que Hume, lo que pretende con estas nociones de libertad y necesidad, al conjuntarlas y hacerlas compatibles, es no excluir la responsabilidad moral del sujeto en cuestión, arguyendo que estos términos son nociones que no solamente son compatibles con la moral, sino que son también, absolutamente, imprescindibles para su conservación.

\section{La noción de causalidad: costumbre y libertad}

Para poder dilucidar el acuerdo entre libertad y necesidad, resulta importante mencionar los cuatro aspectos esenciales que se dan cuando hay causalidad entre dos sucesos A y B, a saber:

I. A y B son contiguos temporal y espacialmente,

2. A y B están en una relación de sucesión temporal,

3. Hay una conjunción constante entre ejemplares de sucesos del tipo A y ejemplares de sucesos del tipo B,

4. La disposición o el hábito según el cual si A y B son contiguos, si están en una secuencia temporal, y si hay una conjunción constante entre As y Bs y las 
satisfacen y, además, si un ejemplar de A no hubiera ocurrido, el correspondiente ejemplar de B tampoco habría ocurrido ${ }^{5}$.

Cuando Hume habla de necesidad física, se refiere expresamente a que las causas, no necesariamente, determinan sus respectivos efectos, es decir, una causa no tiene las condiciones suficientes, ni necesarias, para causar dicho efecto, es por esto que deben cumplir los anteriores requisitos para que se les pueda facultar, en efecto, como causalidad. Otra característica que Hume menciona acerca de la causalidad es que ésta se aplica por igual tanto al mundo exterior como al mundo interior. Hume cree que lo que naturalmente nos lleva a pensar acerca de la existencia de la causalidad, en términos de la naturaleza y acontecimientos físicos, es la misma razón que podemos encontrar para hallar esta causalidad en las acciones humanas.

Llegamos a creer que ciertos eventos físicos están relacionados, ya sea causal o necesariamente, debido a la constante conjunción que hemos observado en el pasado, esta observación y aplicación también se la adjudicamos al comportamiento de los hombres. Hay tanta uniformidad en las acciones humanas como en la naturaleza inanimada; esto no quiere decir que todas las personas sean exactamente las mismas o que actúen de la misma forma, siempre en determinadas circunstancias, simplemente se refiere al hecho de que cada caso representa una uniformidad, tal como se presenta en eventos de objetos inanimados. Y la existencia de igualdades o uniformidades no resulta incompatible en la variedad que pueda existir en asuntos de índole de la conducta humana, ni de la naturaleza inanimada, por ejemplo, "cierta secuencia de eventos hace que un árbol dé manzanas y que otro árbol dé ciruelas, pero no por eso concluimos que los árboles dan sus frutos sin causa alguna" ${ }^{6}$, podemos conceder las diferencias de sus productos finales a su estructura interna.

\footnotetext{
${ }^{5}$ Mota Pinto, Silvio. (2012). El proyecto filosófico de David Hume, Universidad Autónoma Metropolitana, México, p. 255.

${ }^{6}$ Stroud, Op. Cit., p. 202.
} 
La partición que representa, por un lado, la libertad, y por el otro, el determinismo, ha hecho que se susciten diversas preguntas dentro de diferentes ámbitos y que hayan proporcionado definiciones y soluciones ante tales conflictos que se originan, precisamente, por la contraposición de estas dos posturas. Pues bien, el asunto en lo que respecta a la voluntad libre que, se supone, poseemos, sigue representando un sinfín de dudas para el hombre, ya que llega a considerar que sus acciones no dependen completa ni estrictamente de él, sino que existen otros factores que se encuentran fuera de su control e intervienen en la toma de sus decisiones, estos factores pueden ser las leyes físicas, Dios, el destino o los condicionamientos sociales y psíquicos. La dificultad de esta problemática podría radicar en el hecho de que todos nos sentimos libres, sin embargo, no nos sabemos libres, es decir, la disputa se centra en descubrir si esta percepción subjetiva es realmente correcta al afirmar que los hombres, a diferencia del resto del universo, son libres y no están sometidos a leyes universales y deterministas que rigen su comportamiento y sus acciones.

Actualmente la cuestión del libre albedrío resulta muy importante, quizá es por la época en la cual nos encontramos, o debido a los sucesos que han acontecido a lo largo de toda la historia de la humanidad, es decir, las guerras, los conflictos bélicos, las migraciones, los altos índices de violencia a los que se han sometido varios grupos minoritarios, entre otras cosas, es que las personas claman tanto la libertad, incluso cuando la sociedad misma se ha resistido, en diversas ocasiones, a la misma. El filósofo Julius A. Ayer nos explica que a menudo las personas creen que actúan libremente, y que es precisamente esta sensación de libertad a la que muchos filósofos recurren cuando se atiende la cuestión moral, es decir, demostrar que no todas las acciones están causalmente determinadas. Si los filósofos que suponen que un sujeto no puede estar actuando libremente si sus decisiones, o acciones, están causalmente determinadas, "entonces el hecho de que alguien se sienta libre de efectuar, o no efectuar, cierta acción

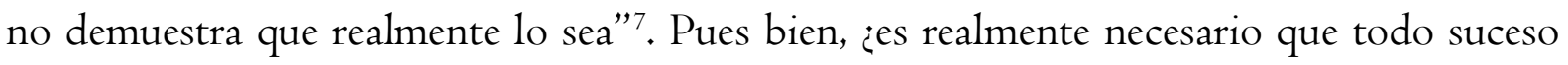

\footnotetext{
${ }^{7}$ Cfr. Ayer, Julius Alfred. (1986). Ensayos filosóficos, Planeta-Agostini, Barcelona.
} 
tenga estrictamente una causa? Si esto fuera cierto, esta regla se aplicaría tanto al comportamiento humano como a cualquier otra cosa. Aunado a esto, también cabría cuestionarse acerca del por qué tenemos que suponer que todo hecho deba tener una causa, ya que ni siquiera la ley de causación universal resulta en una suposición necesaria para el pensamiento científico.

\section{Conclusión}

El científico puede tratar de buscar leyes causales para algunos sucesos y en muchas ocasiones esto tiene éxito, sin embargo, en otras circunstancias tiene que resignar sus resultados a meras leyes estadísticas, pero también existen otros momentos en los cuales concurren hechos que, debido a su estado actual de conocimiento, no se puede enmarcar dentro de ciertas leyes universales o estadísticas. Así podríamos enmarcar un sinfín de ejemplos, de corte cientificista, que arrojan datos concretos y concisos y que no precisamente encajan del todo dentro de una postura determinista o libertarita, incluso podría decirse lo mismo de otras disciplinas, sin embargo, las dos doctrinas han proporcionado fuertes argumentos para afirmar que ambas resultan ser verdaderas. Cada una tiene implicaciones, cuestionamientos, justificaciones y contraargumentos que ponen en duda su legitimidad y pueden compararlos constantemente y ponerlos a prueba, lo que resulta interesante es que ha sobrepasado a la filosofía en el sentido de que otras áreas del conocimiento han querido probar la veracidad, ya sea de la libertad o el determinismo, no solo en cuestiones teóricas, sino también prácticas. 